\title{
Birt-Hogg-Dubé syndrome: an inherited cause of spontaneous pneumothorax
}

\author{
Cameron W. Pierce MD, Peter R. Hull MD PhD, Edmond G. Lemire MD PhD, Darcy D. Marciniuk MD
}

A 56-year-old woman of average build (height $1.6 \mathrm{~m}$, body mass index 26.4) was referred to the respirology clinic for assessment of recurrent, bilateral, spontaneous pneumothoraces of unknown cause. She had her first spontaneous pneumothorax at the age of 28 years and had a total of six spontaneous pneumothoraces, distributed bilaterally, over the next 28 years. Repeated surgical interventions were done, including bullectomies and pleural abrasion. Surgical pathology showed subpleural bullae without generalized emphysematous changes or infiltration of smooth muscle cells. Aside from these acute events, the patient was asymptomatic and had no other respiratory symptoms.

The patient's medical history included postnasal drip, dyslipidemia and hypertension. She had no history of other respiratory illness or abnormalities of the skin, kidneys or central nervous system, and she was a lifetime nonsmoker. The patient's family was of Dutch-Mennonite descent with a strong history of spontaneous pneumothoraces in adulthood, with occurrences in four of seven siblings, the patient's father and two paternal cousins (Figure 1).

The patient's physical examination was normal except for several small, dome-shaped, pale and flesh-coloured papules on her face, the largest of which appeared on her nose (Figure 2). Biopsies of these papules were consistent with fibrofolliculomas (Appendix 1, available at www.cmaj.ca/cgi /content/full/cmaj.092121/DC1). Skin tags were found on the patient's neck.

A radiograph of the patient's chest showed no abnormalities, but multiple thin-walled cysts were seen bilaterally in the lungs on a computed tomography (CT) scan (Figure 3). Tests of our patient's pulmonary function showed that her air flows, lung volumes and diffusing capacity for carbon monoxide were all normal.

A diagnosis of Birt-Hogg-Dubé syndrome was suspected on the basis of clinical and radiographic features, as well as evidence of autoso- mal dominant transmission in the patient's family. The diagnosis was confirmed with a skin biopsy, and a DNA test on a peripheral blood sample showed a heterozygous mutation in the gene encoding folliculin (FLCN).

Subsequent abdominal imaging to assess for renal tumours showed a small $(5 \mathrm{~mm})$ lesion in the patient's right kidney. The lesion is being closely monitored, and has not increased in size on two subsequent CT scans over a period of one year.

\section{Discussion}

Our patient had a personal and family history of recurrent bilateral pneumothoraces despite otherwise normal lung function and no known previous lung disease. In addition to the lesions on her skin and the multiple, thin-walled, pulmonary cysts that were visible on a CT scan, these factors led us to a clinical diagnosis of Birt-HoggDubé syndrome. This diagnosis was confirmed with a skin biopsy and genetic testing.

About $11.5 \%$ of spontaneous pneumothoraces occur in the context of a positive family history ${ }^{1}$ and involve heritable conditions such as $\alpha 1$ antitrypsin deficiency, Marfan syndrome, Ehlers-Danlos syndrome (type IV: vascular type), lymphangioleiomyomatosis (when associated with tuberous sclerosis) and cystic fibrosis. ${ }^{2}$

\section{- KeY POINTS}

- Because a positive family history is found in about $11.5 \%$ of patients presenting with a spontaneous pneumothorax, it is important to consider genetically inherited syndromes among the possible causes.

- Birt-Hogg-Dubé syndrome is a genetic condition with autosomal dominant transmission; classic features include skin lesions, renal tumours and pulmonary cysts that increase the patient's risk for spontaneous pneumothoraces.

- Patients with Birt-Hogg-Dubé syndrome should be regularly checked for renal tumours.

- Identifying a proband with Birt-Hogg-Dubé syndrome provides an opportunity for genetic counselling and screening in the affected individual's family. 


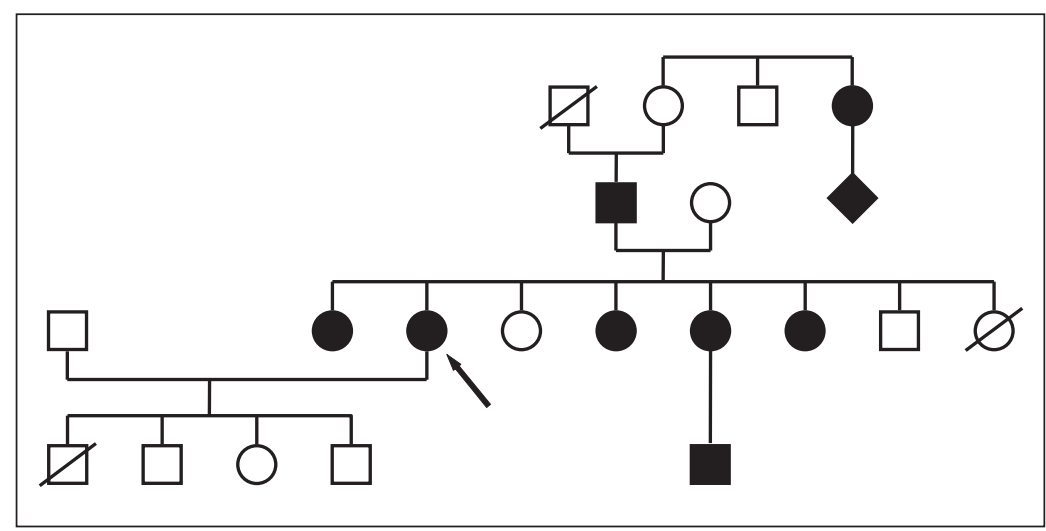

Figure 1: Partial pedigree of a 56-year-old woman with Birt-Hogg-Dubé syndrome (arrow). Filled symbols identify individuals with a confirmed or reported history of spontaneous pneumothorax.

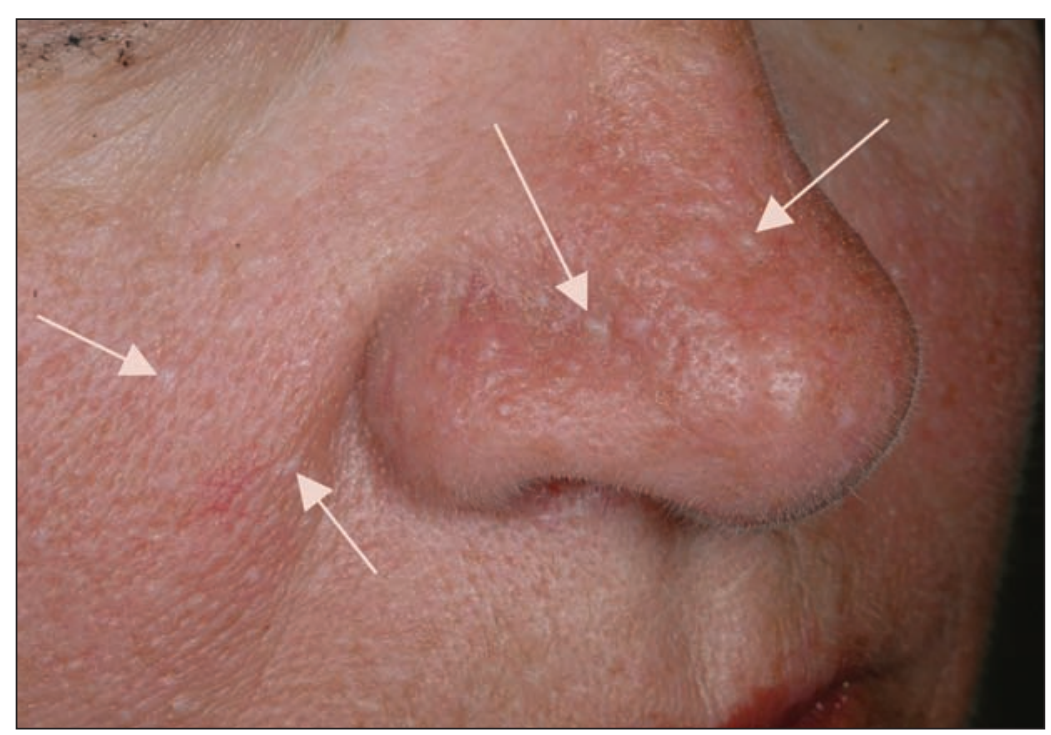

Figure 2: Pale papules (arrows) on the patient's cheeks and nose.

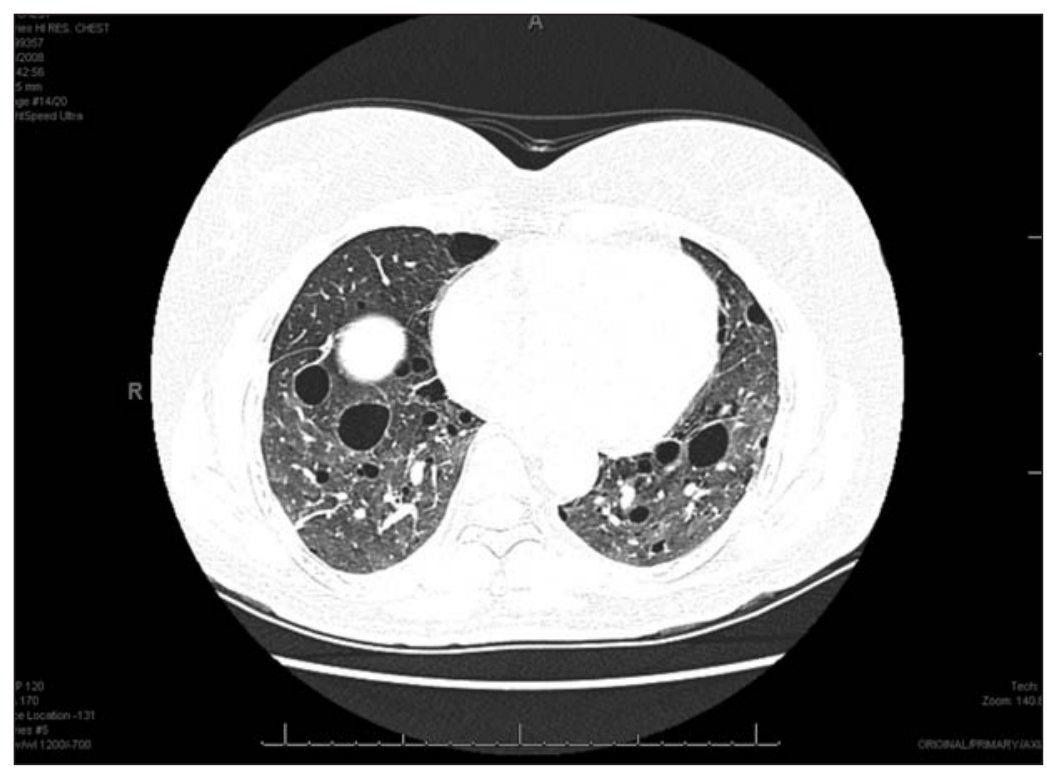

Figure 3: Computed tomography scan of the lower lung fields, showing multiple thin-walled cysts bilaterally.
(Appendix 2, available at www.cmaj.ca/cgi/content /full/cmaj.092121/DC1, outlines the causes of spontaneous pneumothorax, with clinical features and diagnostic testing.) Recently added to this list is Birt-Hogg-Dubé syndrome, a rare genodermatosis that was first described in 1977 in a family from Winnipeg, Manitoba, on the basis of skin lesions that showed a pattern of autosomal dominant inheritance. ${ }^{3}$ The cardinal cutaneous features of this syndrome are follicular hamartomas - fibrofolliculomas, trichodiscomas and acrochordon-like growths — which usually become apparent only in the third or fourth decades of life. ${ }^{4,5}$ Fibrofolliculomas and trichodiscomas present as multiple, small, pale or skin-coloured, dome-shaped papules. They are usually seen on the face but are sometimes found on the neck and upper trunk. Acrochordon-like growths that are simple-togloboid in shape are found on the neck, trunk and arms and in the axillae. Although the diagnosis has historically been based on the presence of 5-10 fibrofolliculomas, one of which should be confirmed histologically, ${ }^{4}$ there is increasing reliance on nondermatologic findings and genetic testing in the diagnosis of Birt-Hogg-Dubé syndrome.

In addition to fibrofolliculomas and related skin findings, Birt-Hogg-Dubé syndrome is associated with cystic lung disease, spontaneous pneumothoraces, and benign and malignant renal tumours. ${ }^{2,4}$ Cystic lung disease is estimated to occur in $84 \%$ of patients with Birt-Hogg-Dubé syndrome, and spontaneous pneumothoraces occur in about $38 \%$ of patients. ${ }^{6}$ Both conditions may occur without associated dermatologic or renal findings. ${ }^{1}$ Plain chest radiography may appear normal, and CT scans are often required to detect pulmonary cysts. The prevalence of renal tumours such as oncocytomas, chromophobe renal cell carcinomas, clear cell carcinomas and papillary renal cell carcinomas is about 34\% among patients with Birt-HoggDubé syndrome.,

\section{Genetics}

The genetic locus responsible for the syndrome has recently been mapped to chromosome 17 (17p11.2) and the gene encoding folliculin (FLCN). The folliculin protein is 579 amino acids long. It is expressed in stromal cells, the skin and its appendages, the distal nephron and type I pneumocytes. ${ }^{6}$ Although the exact function of folliculin is not known, there is some evidence to support its role as a tumour suppressor. More than 50 unique germline mutations in FLCN have been reported, most of which are frameshift or nonsense mutations predicted to result in premature truncation of the protein. Mutation analysis can detect mutations in nearly $90 \%$ of patients clinically suspected of having Birt-Hogg-Dubé syndrome. ${ }^{4,6}$ In our 
patient, amplification of exons 4-14 by polymerase chain reaction showed a deletion of a single thymine residue at nucleotide 59 of the $F L C N$ gene (c.59delT) resulting in the premature truncation of the protein at amino acid 54 .

\section{Management}

Caring for patients with Birt-Hogg-Dubé syndrome includes an assessment at baseline, ongoing surveillance and treatment of symptoms as needed. High-resolution CT scans of the chest will help identify pulmonary cysts. Subsequent pneumothoraces are treated in the same way as other spontaneous pneumothoraces, with particular consideration given to interventions that will reduce the risk of recurrence.

Patients with Birt-Hogg-Dubé syndrome should have a careful dermatologic examination, and any suspicious lesions should be biopsied. Because regular imaging of the abdomen is important for the surveillance of renal tumours, magnetic resonance imaging is preferred over CT scans to minimize the patient's exposure to radiation. Complementary use of renal ultrasound may help clarify the nature of small lesions. It may be possible to follow small $(<3.0 \mathrm{~cm})$ renal lesions before considering surgery; consultation with a urologist is appropriate..$^{5}$

This report highlights the importance of considering genetically inherited syndromes when a patient presents with recurrent pneumothoraces, especially when there is a positive family history. Meaningful interaction between multiple subspecialty teams facilitates the correct investigations and diagnosis. Moreover, establishment of the diagnosis enables the appropriate screening for associated malignant diseases that would not otherwise have been considered.

\section{References}

1. Graham RB, Nolasco M, Peterlin B, et al. Nonsense mutations in folliculin presenting as isolated familial spontaneous pneumothorax in adults. Am J Respir Crit Care Med 2005;172:39-44.

2. Toro JR, Pautler S, Stewart L, et al. Lung cysts, spontaneous pneumothorax, and genetic associations in 89 families with Birt-Hogg-Dubé syndrome. Am J Respir Crit Care Med 2007; 175:1044-53.
3. Birt A, Hogg GR, Dubé WJ. Hereditary multiple fibrofolliculomas with trichodiscomas and acrochordons. Arch Dermatol 1977;113: 1674-7.

4. Toro JR, Glenn G, Duray P, et al. Birt-Hogg-Dubé syndrome: a novel marker of kidney neoplasia. Arch Dermatol 1999;135: 1195-202.

5. Toro J. Birt-Hogg-Dubé syndrome. GeneReviews. GeneTests: Medical Genetics Information Resource [database]. Seattle (WA): University of Washington; 1993-2010. Available: www .genetests.org (accessed 2009 July 28).

6. Toro JR, Wei M, Glenn G, et al. BHD mutations, clinical and molecular genetic investigations of Birt-Hogg-Dubé syndrome: a new series of 50 families and a review of published reports. $J$ Med Genet 2008;45:321-31.

Competing interests: Darcy Marciniuk is a consultant for AstraZeneca, Boehringer-Ingelheim, GlaxoSmithKline, Health Quality Council, Novartis, Nycomed, Pfizer, the Public Health Agency of Canada and Saskatoon Health Region; he has received grants from AstraZeneca, Boehringer-Ingelheim, the Canadian Agency for Drugs and Technology in Health, the Canadian Institutes of Health Research, GlaxoSmithKline, the Lung Association of Saskatchewan, Novartis, the Royal University Hospital Foundation, the Saskatchewan Ministry of Health and Schering Plough; he has received speaker fees from AstraZeneca, Boehringer-Ingelheim, GlaxoSmithKline, the Lung Association of Saskatchewan and Pfizer; and he has developed educational presentations for AstraZeneca, Boehringer-Ingelheim and Pfizer.

Affiliations: From the Departments of Respirology (Pierce, Marciniuk), Dermatology (Hull) and Genetics (Lemire), University of Saskatchewan, Saskatoon, Sask.

Contributors: All of the authors saw the patient in consultation, derived the clinical data, helped draft the manuscript, revised it for important intellectual content and approved the final version submitted for publication.

The section Cases presents brief case reports that convey clear, practical lessons. Preference is given to common presentations of important rare conditions, and important unusual presentations of common problems. Articles start with a case presentation (500 words maximum), and a discussion of the underlying condition follows (1000 words maximum). Generally, up to five references are permitted and visual elements (e.g., tables of the differential diagnosis, clinical features or diagnostic approach) are encouraged. Written consent from patients for publication of their story is a necessity and should accompany submissions. See information for authors at www.cmaj.ca. 